\title{
L'Etat et la Demande de Sécurité dans les Sociétés Contemporaines
}

\author{
par Raymond Barre *
}

L'activité économique, comme toute activité humaine, est caractérisée par l'incertitude et exposée au risque. Tout calcul économique tend à limiter le risque ou à en atténuer les conséquences désagréables éventuelles.

Le comportement des agents économiques est en effet inspiré dans une importante mesure par le désir de sécurité. L'ampleur de l'incertitude et la crainte de risques trop élevés peuvent être un frein à l'action. Aussi le calcul économique doit-il déterminer un point d'équilibre entre risque et sécurité.

L'activité économique se déroule dans un environnemenet déterminé par les mentalités individuelles et collectives, les règles sociales, l'état de la technique, la politique de l'Etat. Les conditions dans lesquelles se présente le risque et dans lesquelles il peut être assumé sont donc variables. La société est, elle aussi, conduite à rechercher un point d'équilibre entre risque et sécurité et à mettre en œuvre des mécanismes collectifs de protection et de couverture des risques.

A un faible niveau de développement de l'économie, l'épargne individuelle apparaît comme la première manifestation de l'assurance contre les risques : elle est souvent en nature et constitue une garantie de survie. Dans une économie monétarisée, les encaisses liquides de transaction et de précaution permettent de faire face à l'imprévu.

Mais, dans les économies modernes, des institutions spécifiques ont pour rôle d'apporter aux individus et aux entreprises la couverture des risques, contre lesquels ils souhaitent s'assurer.

La demande d'assurance n'a cessé de se diversifier : la complexité croissante des activités entraîne une multiplication des risques; l'élévation du revenu permet d'affecter une part plus importante des ressources à la satisfaction du besoin d'assurance. L'offre d'assurance s'est efforcée d'y répondre, tant pour les individus que pour les entreprises.

Mais l'évolution des mentalités et des sociétés a conduit à un phénomène aujourd'hui fondamental dans les sociétés industrialisées : une intervention étendue de l'Etat, qui met en œuvre des systèmes d'assurance fondés sur le principe de la solidarité nationale.

* Professeur à l'Université de Paris 2. Ancien Président de l'Association de Genève et ancien Premier Ministre du Gouvernement Français. 
Ce sont les aspects et les conséquences de ce phénomène que je voudrais examiner rapidement ici. Il soulève en effet des problèmes d'une grande importance :

- problème des domaines couverts par l'assurance publique ;

- problème du financement de cette assurance et de ses effets sur l'économie et les finances publiques ;

- problème des rapports entre les institutions publiques d'assurance et les institutions privées qui ont rempli jusqu'ici ce rôle.

Mais, au-delà de ces problèmes, il y a une question centrale qui paraît, à mes yeux, se poser déjà dans beaucoup de pays. Je la présenterai de la manière suivante :

Nous vivons dans un monde où l'incertitude est croissante et les risques de plus en plus étendus. Dans le même temps nous observons une tendance croissante à la demande de sécurité. Dans quelle mesure la demande de sécurité peut-elle être satisfaite par l'Etat, sans freiner ou empêcher les adaptations que rend inévitables et nécessaires le profond changement du monde?

Cette question concerne en fin de compte un choix de société. Allons-nous vers une société d'assistance, où l'Etat assume les risques des citoyens, ou entendons-nous sauvegarder une société de liberté et de responsabilité, où l'assomption des risques reste répartie entre l'Etat, des institutions privées d'assurance et les citoyens eux-mêmes ? Autrement dit, dans la société de demain, l'assurance doit-elle être une œuvre collective ou une réalisation collectiviste?

Il suffit d'observer les sociétés contemporaines pour mesurer l'importance de cette question ; celle-ci prend un relief d'autant plus accusé qu'elle se pose en une période de crise, qui semble durable. Les réponses restent confuses et hésitantes; à cet égard l'expérience Reagan aux Etats-Unis comme l'expérience socialiste en France représentent deux types opposés et symboliques de réponse, qu'il n'est pas encore possible d'apprécier de façon définitive, même si, dans un cas comme dans l'autre, le succès ne semble pas certain.

Au-delà de ces situations concrètes, je voudrais ici présenter quelques réflexions sur ce que j'appellerai le besoin de sécurité dans les sociétés contemporaines et les moyens d'y faire face dans le cadre d'une société de progrès.

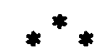

L'aspiration à la sécurité, longtemps individuelle, est devenu une aspiration collective de plus en plus intense à partir de la Grande Dépression des « années 30 ».

Le spectacle d'individus sans emploi et sans revenu, ne pouvant couvrir les risques fondamentaux de l'existence, a favorisé la recherche de mécanismes permettant de garantir aux individus, dans les circonstances les plus difficiles de l'existence, une aide de la collectivité. Avec Beveridge naissait, au lendemain de la seconde guerre mondiale, la conception moderne de la Sécurité Sociale dont l'application généralisée a conduit au * Welfare State ». Cette application a été largement facilitée et favorisée par la grande période de prospérité qu'a connue le monde occidental depuis 1950 jusqu'aux années 1970. La croissance rapide de l'économie favorisait la formation régulière d'un «surplus» qui permettait à la fois l'augmentation des revenus individuels et un ample processus de redistribution des revenus. Tous les gouvernements, sous la pression de 
leurs opinions ou dans une conception généreuse de leur action, ont assuré une couverture croissante des risques et cherché à donner aux citoyens une sorte de garantie générale de sécurité.

Les systèmes de sécurité sociale assurent les risques liés pour les individus à :

- la maladie ;

- la vie familiale ;

- la vieillesse.

D'autres systèmes de protection sociale couvrent le risque du chômage.

La protection, accordée d'abord aux salariés, a eu tendance à se généraliser à l'ensemble des citoyens.

De plus, le risque économique lié à l'activité d'entreprise, et longtemps supporté par les entreprises elles-mêmes ou par des institutions d'assurance privée, tend dans certaines circonstances à être pris en charge par des mécanismes étatiques. L'Etat développe l'assurance-crédit à l'exportation ; pour parer à l'instabilité monétaire internationale, il met en place des garanties de change. Lorsqu'une entreprise, qui tient une place importante dans l'économie nationale ou dans l'économie régionale, éprouve des difficultés, l'Etat lui accorde une aide, sous forme de subventions, commandes ou prêts avantageux, au nom de considérations variées, dont la plus importante est l'emploi. La logique de l'économie privée, fondée sur l'assomption du risque, cède progressivement le pas à une autre logique : celle de l'économie publique. Enfin, dans certains cas, les revenus d'une catégorie entière d'agents économiques - les agriculteurs - sont garantis contre les aléas des récoltes et les variations des prix agricoles.

Cette extension considérable de la protection des risques comporte un coût élevé pour l'économie.

Elle entraîne tout d'abord une explosion des dépenses publiques et plus particulièrement des dépenses sociales. Depuis 1965 jusqu'à la fin des années 1970, le ratio dépenses publiques / P.I.B. indique une hausse de plus de 10 points de pourcentage pour les principaux pays industrialisés, dont en particulier le Royaume-Uni, l'Allemagne, la France, l'Italie, les Pays-Bas, la Norvège, la Suède et la Suisse.

L'augmentation des dépenses entraîne en second lieu un accroissement massif de l'impôt sur le revenu et des cotisations de sécurité sociale. La pression fiscale de l'ensemble des pays de l'OCDE est passée de $28 \%$ en 1965 à $36,6 \%$ en 1980.

Le fardeau pesant sur l'économie productive au titre du financement de la sécurité a plusieurs conséquences :

- une hausse plus rapide des prix, l'inflation étant un moyen de rejeter sur l'ensemble des consommateurs la charge pesant sur les agents productifs. A la fin des annnées 1940, Colin Clark avait soutenu la thèse selon laquelle l'inflation deviendrait inévitable lorsque la pression fiscale dépasserait $25 \%$. Nous sommes aujourd'hui bien au-delà ;

— un accroissement du chômage dû à l'augmentation trop vive des coûts de production, qui incorporent les cotisations sociales payées par les entreprises ;

- une rigidité croissante de l'économie dont les ressources deviennent moins mobiles du fait des garanties de revenu, et qui sont utilisées de manière moins efficace ; 
— un affaiblissement de l'incitation au travail et à l'épargne ;

- une propension plus élevée chez les agents économiques à se livrer à des activités « souterraines » échappant à l'impôt.

La croissance économique, qui est indispensable pour financer les dépenses de sécurité, se trouve ainsi ralentie. L'économie s'engage sur la voie de la stagnation, accompagnée d'inflation et de chômage. Un processus cumulatif de déséquilibre entre en jeu : l'accroissement des charges provoque un accroissemenet du chômage, qui entraîne à son tour un accroissement des dépenses publiques et une nouvelle poussée d'inflation.

Or, cette évolution économique défavorable s'effectue à l'heure actuelle, dans un monde où se présentent des risques collectifs d'une grande ampleur :

— risques liés à une forte instabilité monétaire, qu'ont provoquée depuis 1971 la disparition du système monétaire international et l'avènement du régime des changes flottants ;

- risques liés à la vulnérabilité du système financier international, due à l'endettement massif de certains pays développés ou en voie de développement et aux dangers du recyclage des capitaux internationaux;

- risques liés à une intensification massive de la concurrence internationale, qui appelle une adaptation profonde des économies industrialisées, et, pour cela, une plus grande mobilité des ressources et une plus grande ardeur à l'effort productif.

L'esprit de sécurité tend à inspirer diverses réactions à ces risques collectifs :

- la recherche d'une garantie contre les risques monétaires par l'indexation sur le plan économique intérieur, par la «dévaluation concurrentielle» sur le plan des relations de change;

- le recours à des mesures de protection, pour sauvegarder l'activité économique et l'emploi.

Il apparaît donc que la recherche constante d'un équilibre entre le risque et la sécurité, dont dépendent la vigueur et le progrès de l'économie, se trouve affectée par l'importance croissante accordée à la satisfaction du besoin de sécurité des individus. Le danger se trouve dans la baisse de la productivité globale de l'économie, le recul du niveau de vie et une régulation de plus en plus étendue des activités économiques par l'Etat.

Beveridge proclamait: «Full employment in a free society». Nous risquons d'aboutir à une autre situation : « unemployment in a closed and controlled society » . Pent-on arrêter une telle évolution ou celle-ci est-elle irréversible ?

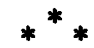

L'observation des sociétés contemporaines conduit à l'heure actuelle à deux constatations :

- d'une part, les individus, qui ont bénéficié d'une croissance régulière de leur revenu et d'une amélioration constante de leur niveau de vie au cours des vingt dernières années, ne paraissent pas prêts à accepter une régression économique ; ils savent 
que les pays où l'activité économique et sociale est étroitement réglementée sont ceux où le niveau de vie est le plus faible, même si la sécurité de l'emploi et du revenu et la protection sociale y sont amplement assurés ;

- d'autre part, ils n'admettraient pas des modifications au régime de protection sociale, qui apparaîtraient comme un retour au passé ou comme un recul.

Le problème est donc, du point de vue de la politique économique et sociale, de trouver un point d'équilibre auquel le niveau de protection sociale demeure compatible avec le respect des équilibres économiques fondamentaux et la sauvegarde des incitations à la croissance et au progrès. Il s'agit d'un problème de "Risk management » au niveau de la collectivité nationale.

Je ne saurais, dans le cadre de cet exposé, traiter de façon complète cette question. Je me limiterai à une esquisse des solutions, qui me paraissent politiquement et socialement praticables.

1. L'Etat doit, en premier lieu, s'efforcer de réduire les risques économiques collectifs qui pèsent sur les citoyens.

Le plus important de ces risques - celui qui a les effets les plus étendus sur la collectivité - est l'inflation. L'inflation provoque le chômage, affaiblit l'épargne, réduit le pouvoir d'achat des revenus et des pensions de retraite, compromet la valeur internationale de la monnaie.

La lutte contre l'inflation ne peut se réduire à un contrôle, même draconien, de la masse monétaire. L'expérience des Etats-Unis comme celle d'autres pays, montre que l'instrument monétaire perd son efficacité s'il n'est pas utilisé conjointement avec l'instrument budgétaire. Le drame des économies contemporaines est l'ampleur du déficit budgétaire, qui a pour résultat d'accroître la pression du Trésor sur le marché des capitaux et de provoquer une forte hausse des taux d'intérêts qui freine l'investissement.

Le ralentissement durable de la hausse des prix, le retour à une croissance économique soutenue par l'investissement, ne seront obtenus dans l'économie occidentale que par la mise en œuvre d'une politique budgétaire tendant à :

a) mettre un terme à la progression régulière et rapide des dépenses publiques, qui devraient croître moins vite que le Produit National Brut en valeur ;

b) ramener le rythme de progression des dépenses sociales à celui du Produit National Brut ;

c) utiliser les résultats obtenus dans la maîtrise des dépenses publiques et sociales pour réduire les prélèvements sur le revenu des particuliers et les charges pesant sur les entreprises.

La situation générale des économies occidentales impose désormais de réduire les dépenses pour alléger les impôts au lieu d'accroître systématiquement les impôts pour couvrir les dépenses.

2. L'Etat doit, en second lieu, limiter l'extension de la couverture des risques par la solidarité nationale et faciliter, à côté de la protection par la collectivité des risques fondamentaux pesant sur les individus, le développement de mécanismes de couverture des risques fondés sur l'assurance de groupe ou sur l'assurance individuelle. 
L'idée, qui justifie une telle orientation, est qu'au niveau élevé de revenu existant dans les sociétés industrialisées, la participation de l'individu aux mécanismes de protection sociale peut être accrue par rapport à celle assumée jusqu'ici par l'Etat. Il est évident que l'intervention de l'Etat resterait indispensable en faveur des individus les moins favorisés.

Trois exemples peuvent illustrer cette orientation :

1. En ce qui concerne l'indemnisation du chômage, les mécanismes financiers mis en œuvre dans certains pays permettent au salarié ayant perdu son emploi de recevoir pendant une durée assez longue une allocation presque équivalente au salaire précédemment perçu ; le chômeur n'est par ailleurs assujetti à aucune obligation en ce qui concerne l'acceptation d'un nouvel emploi.

Le recours abusif à ces dispositions a conduit d'une part à une situation où, en période de chômage, des employeurs ne trouvent pas la main-d'œuvre dont ils ont besoin, d'autres part, au développement du « travail noir ».

La nécessité apparaît donc de corriger ces déviations par deux moyens :

- d'une part, une forte dégressivité de l'indemnité de chômage sur la période pendant laquelle elle est versée ; c'est la voie dans laquelle s'était engagée la France en 1979 après qu'une indemnisation de $90 \%$ du salaire antérieurement perçu ait été appliquée pendant plusieurs années ;

- d'autres part, l'obligation faite au chômeur d'accepter, à partir d'un certain délai, un emploi qui lui est offert ; c'est la voie sur laquelle s'est tout récemment engagé le gouvernement de l'Allemagne Fédérale.

2. En ce qui concerne l'assurance-maladie, il apparaît de plus en plus souhaitable, en plus d'une politique d'économies justifiée, d'agir sur les conditions de remboursement des dépenses et d'accroître le degré de participation des individus au financement de ces dépenses.

Une formule déjà utilisée est celle du « ticket modérateur » qui peut varier selon les catégories de prestations.

Une autre formule serait une modulation des prestations remboursées en fonction du revenu ou, pour utiliser une autre expression, une assurance sociale personnalisée.

Dans ces deux cas, les risques les plus importants continueraient à être entièrement couverts par la collectivité. Dans un cas comme dans l'autre, il appartiendrait à l'individu de financer par une cotisation spéciale une couverture plus étendue qu'il souhaiterait obtenir. C'est ainsi que l'on a constaté, au cours de ces dernières années, une croissance de l'assurance maladie volontaire, complémentaire de la sécurité sociale.

3. En ce qui concerne les régimes de retraite, le problème qui est souvent posé est celui du choix entre le système de répartition et le système de capitalisation. Mais des études récentes ont montré qu'il n'est pas justifié d'opposer capitalisation à répartition, mais de rechercher pour les diverses catégories d'individus quelle part de financement de la retraite peut être assurée par l'un et l'autre système.

Le niveau des transferts des cotisations aux retraités par répartition peut être déterminé par l'Etat en fonction de considérations démographiques, économiques et financières; le complément de retraite que peuvent souhaiter certains individus sera alors assuré par un mécanisme de capitalisation. 
Cette capitalisation peut être individuelle, du moins pour ceux qui en ont les moyens financiers.

Elle peut être collective par l'intermédiaire d'institutions de capitalisation, qui peuvent offrir des procédures d'épargne contractuelle intéressantes pour l'individu et aident à la formation d'une épargne longue nécessaire à l'économie.

L'analyse qui précède montre quelles perspectives peuvent s'offrir aux systèmes de protection sociale dans les sociétés modernes.

Il est clair que les Etats sont désormais contraints de fixer une limite à la progression des dépenses de couverture des risques : même s'ils ne le voulaient pas, les conditions économiques objectives les y contraindraient, sauf à changer le type d'organisation de l'économie et de la société.

Pour satisfaire le besoin de sécurité des individus, il apparaît donc utile, d'une part, de laisser une part plus grande à la responsabilité individuelle dans le choix du niveau de protection souhaitable et des mécanismes de protection utilisables, d'autre part de développer des mécanismes complémentaires et non étatiques de protection sociale.

Les compagnies d'assurance peuvent jouer à cet égard un rôle très important tant dans l'offre de produits adaptés, susceptible de répondre à la demande des individus ou des groupes que dans la gestion des ressources d'épargne qu'ils collectent au service de l'économie nationale.

La voie de l'avenir ne réside pas dans un choix entre logique publique et logique privée de la protection sociale, mais dans une action collective qui associe le secteur public et le secteur privé, et qui tient compte à la fois des avantages et des limites que chacun d'eux présente.

Telle doit être l'une des formes d'innovation sociale, dont ont besoin nos sociétés pour leur stabilité et leur développement. 